\title{
Informationen zum Fortbildungszertifikat
}

Das Fortbildungszertifikat Phlebologie (DGP) der Deutschen Gesellschaft für Phlebologie bietet die Möglichkeit eine qualifizierte und fortlaufende Fortbildung im Bereich Phlebologie $\mathrm{zu}$ dokumentieren und dies auch auf dem Praxisschild auszuweisen.

Die ersten Fortbildungszertifikate Phlebologie wurden 2013 im Rahmen der Jahrestagung in Hamburg vergeben und inzwischen sind es circa 130 . Wir blicken also auf 5 erfolgreiche Jahre zurück

Die Phlebologie ist ein Bereich, dessen Inhalte in den letzten 20 Jahren durch viele neue Entwicklungen geprägt waren. Die steigende Zahl neuer Mitglieder der DGP und die Resonanz auf phlebologische Fortbildungsveranstaltungen zeigen das wachsende Interesse insbesondere auch junger Kolleginnen und Kollegen.

Seit 1992 besteht die Möglichkeit des Erwerbs der Bereichsbezeichnung Phlebologie im Rahmen des Weiterbildungsprogramms der Ärztekammern. Sie ist an eine 18-monatige Weiterbildung in einer anerkannten Weiterbildungsstätte in einer Klinik oder Praxis und eine Prüfung bei der jeweiligen Ärztekammer gebunden. In der Regel geschieht dies vor der Niederlassung in eigener Praxis. Danach findet die Weiterbildung nur in Ausnahmefällen statt und auch die Zahl der anerkannten Weiterbildungsstätten ist begrenzt.

Neben diesem offiziellen Weiterbildungsweg und den von der Ärztekammer anerkannten Phlebologen gibt es aber zahl- reiche Kolleginnen und Kollegen, die eine wichtige schwerpunktmäßige phlebologische Tätigkeit in Klinik oder Praxis ausüben, ohne die Möglichkeit gehabt zu haben den „Phlebologen“ zu erwerben. Sie haben ihr Wissen und ihre Erfahrungen meist durch Fortbildungsaktivitäten und Hospitationen erworben. Darunter sind auch viele Kolleginnen und Kollegen aus anderen Fachgruppen vertreten wie Gefäßchirurgen, Angiologen und Dermatologen, in denen phlebologische Weiterbildungsinhalte etabliert sind, die aber die zusätzliche Weiterbildung Phlebologie aus verschiedenen Gründen nicht abgeschlossen haben.

Speziell für diese Kolleginnen und Kollegen hat die DGP das Fortbildungszertifikat Phlebologie (DGP) etabliert, das eine adäquate Qualifikation in der Phlebologie dokumentiert und darüber hinaus auch eine kontinuierliche Fortbildung in diesem Bereich. Bereichsbezeichnete Phlebologen können damit zusätzlich ihre ständige Fortbildung in der Phlebologie belegen, was mit der Zusatzbezeichnung alleine nicht möglich ist. Der Nachweis der schwerpunktmäßigen phlebologischen Tätigkeit orientiert sich an den Inhalten der Weiterbildungsordnung Phlebologie. Das FBZ Phlebologie ist nach Anmeldung bei der Ärztekammer auf dem Praxisschild führbar.

Die Kriterien zum Erwerb des Fortbildungszertifikats Phlebologie der Deutschen Gesellschaft für Phlebologie sind:
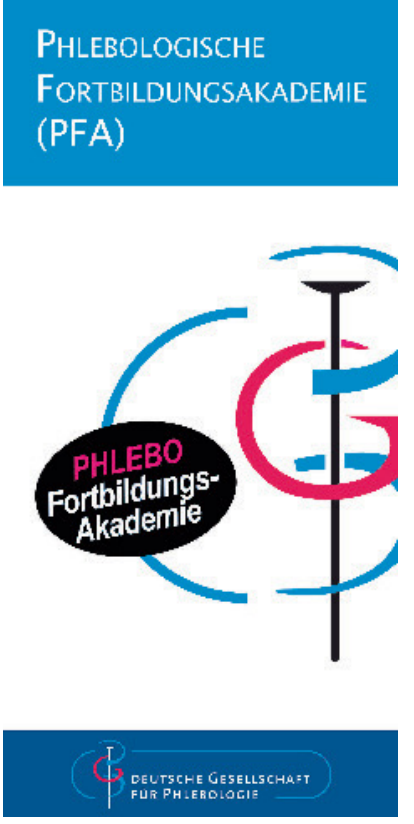

Ärzte mit anerkannter Weiterbildung

Phlebologie erwerben das Zertifikat, wenn sie folgende Voraussetzungen erfüllen:

- Mitgliedschaft in der DGP

- Nachweis von 20 Fortbildungspunkten im phlebologischen Bereich je Jahr in den letzten 3 Jahren (z. B. Teilnahme an offiziellen Fortbildungsaktivitäten der DGP und / oder von der DGP offiziell anerkannten Fortbildungsveranstaltungen)

- Nachweis Zusatzbezeichnung Phlebologie

- Nachweis einer schwerpunktmäßigen phlebologischen Tätigkeit

Ärzte ohne anerkannte Weiterbildung Phlebologie, aber mit Facharztbezeich-

Übersicht der Anforderungen an den Erwerb des Fortbildungszertifikates Phlebologie

\begin{tabular}{|c|c|c|c|c|}
\hline $\begin{array}{l}\text { Anforderungen an den Erwerb } \\
\text { des Fortbildungszertifikates } \\
\text { Phlebologie für }\end{array}$ & $\begin{array}{l}\text { Ärzte mit Zusatz- } \\
\text { bezeichnung } \\
\text { Phlebologie }\end{array}$ & $\begin{array}{l}\text { Ärzte ohne Zusatzbezeichnung } \\
\text { Phlebologie aber mit Facharzt } \\
\text { für Angiologie, Gefäßchirurgie } \\
\text { oder Dermatologie }\end{array}$ & $\begin{array}{l}\text { Ärzte ohne Zusatzbezeichnung } \\
\text { Phlebologie und ohne Facharzt } \\
\text { für Angiologie, Gefäßchirurgie } \\
\text { oder Dermatologie }\end{array}$ & $\begin{array}{l}\text { Erneuerung des } \\
\text { Zertifikats nach } \\
3 \text { Jahren }\end{array}$ \\
\hline Mitglied in der DGP & Ja & Ja & Ja & $\mathrm{Ja}$ \\
\hline $\begin{array}{l}60 \text { Fortbildungspunkte Phlebologie } \\
\text { in den letzten } 3 \text { Jahren }\end{array}$ & Ja & Ja & Ja & Ja \\
\hline $\begin{array}{l}2 \text { Jahre schwerpunktmäßige Tätig- } \\
\text { keit Phlebologie }\end{array}$ & Ja & $\mathrm{Ja}$ & Ja & Nein \\
\hline Basiskurs Phlebologie & Nein & Nein & $\mathrm{Ja}$ & Nein \\
\hline 2 Hands-on Kurse Phlebologie & Nein & Nein & $\mathrm{Ja}$ & Nein \\
\hline Kolloquium & Nein & Ja & Ja & Nein \\
\hline
\end{tabular}


nung Dermatologie, Gefäßchirurgie oder Angiologie erwerben das Zertifikat, wenn sie folgende Voraussetzungen erfüllen:

- Mitgliedschaft in der DGP

- Nachweis von 20 Fortbildungspunkten im phlebologischen Bereich je Jahr in den letzten 3 Jahren (z.B. Teilnahme an offiziellen Fortbildungsaktivitäten der DGP und / oder von der DGP offiziell anerkannten Fortbildungsveranstaltungen)

- Nachweis einer schwerpunktmäßigen phlebologischen Tätigkeit

- Ablegen eines Kolloquiums zu phlebologischen Inhalten
Ärzte ohne abgeschlossene Weiterbildung Phlebologie und ohne Facharztbezeichnung Dermatologie, Gefäßchirurgie oder Angiologie erwerben das Zertifikat, wenn sie folgende Voraussetzungen erfüllen:

- Mitgliedschaft in der DGP

- Nachweis von 20 Fortbildungspunkten im phlebologischen Bereich je Jahr in den letzten 3 Jahren (z.B. Teilnahme an offiziellen Fortbildungsaktivitäten der DGP und / oder von der DGP offiziell anerkannten Fortbildungsveranstaltungen)

- Nachweis einer schwerpunktmäßigen phlebologischen Tätigkeit Teilnahme am Grundkurs Phlebologie und min-

destens 2 von der DGP anerkannten Hands-on Workshops

- Ablegen eines Kolloquiums zu phlebologischen Inhalten

Das Zertifikat ist für 3 Jahre gültig. Es wird nach Vorlage von mindestens 60 Fortbildungspunkten im phlebologischen Bereich in den vorangegangenen 3 Jahren auf Antrag erneuert.

Die Unterlagen werden beim Sekretariat der DGP eingereicht. Die Bearbeitungsgebühr beträgt $150 €$ für den Erstantrag. Weitere Informationen finden Sie auf der Website der DGP www.phlebology.de

Dr. Erika Mendoza

\section{Bringen Sie sich ein! Ehrenamt in der Phlebologie}

Unsere Gesellschaft hat viele Aufgaben, im Wesentlichen die Unterstützung der täglichen Arbeit zur wissenschaftlichen Aufarbeitung unseres Fachbereichs, die Fortbildung und die Sicherung eines hohen Standards bei der Versorgung der Patienten. Somit sind wir alle täglich darin involviert.

Viele unserer Mitglieder beteiligen sich darüber hinaus an den verschiedenen Aufgaben unserer Fachgesellschaft. Nicht nur direkt im Vorstand und Beirat, sondern darüber hinaus in vielen anderen Bereichen: In der Schriftleitung der Zeitschrift Phlebologie, im wissenschaftlichen, in vielen
Arbeitsgemeinschaften, in Form von Fortbildungsveranstaltungen und - besonders arbeitsintensiv - in den Leitlinienkommissionen und in der Vorbereitung unserer gemeinsamen Jahrestagungen.

All diese Aktivitäten werden selbstverständlich ehrenamtlich ausgeführt, sprich ohne Honorar. Demgegenüber bekommt man durch sein Engagement andere Entschädigungen, wie das Kennenlernen von weiteren Interessierten und ähnlich gesinnten Menschen, das gemeinsame Erarbeiten von Projekten und dabei das Erweitern des eigenen Horizonts und nicht zuletzt die
Freude, zu sehen, wenn ein Projekt gut abgeschlossen wird, wenn wir Fortschritte machen. Schöne Beispiele hierzu sind die Freigabe aller Kosten rund um die Kompressionsstrümpfe Klasse I, auch in der An- und Ausziehhilfe für Pflegebedürftige oder die Erfolgsgeschichte unseres Fortbildungszertifikats Phlebologie.

Nicht nur die Zahl der Mitglieder ist erfreulicherweise gestiegen, sondern auch unsere Aktivitäten. Jederzeit sind wir auf Unterstützung durch unsere Mitglieder angewiesen. Sprechen Sie uns an - telefonisch, per Mail oder persönlich auf den Tagungen.

Dr. Erika Mendoza

\section{Brief an die Delegierten des 121. Deutschen Ärztetags:}

Beschluss der Bundesärztekammer senkt die Zahl von interdisziplinären Gefäßspezialisten für Venenkrankheiten! Die Zusatzweiterbildung Phlebologie darf nicht abgeschafft werden!

Sehr geehrte Damen und Herren,

die Deutsche Gesellschaft für Phlebologie protestiert scharf gegen die Pläne der Bundesärztekammer, die Zusatzweiterbildung Phlebologie abzuschaffen und empfindet 